Francesca Minerva, Phd.D

Post-doctoral fellow, CAPPE, University of Melbourne.

e-mail: francesca.minerva@unimelb.edu.au

Address: University Park, University of Melbourne, Old Quad Building, 3010 Melbourne (VIC) Australia

Telephone: Mobile-0451-306580, office 03834-49951 


\section{Neuroetica, a look at the development of the Italian debate on neuroethics.}

Neuroetica, science of the brain, philosophy and free will [1] is a collection of eight papers edited by Andrea Lavazza, scholar of Philosophy of Mind and Cognitive Science and Giuseppe Sartori, Professor of Cognitive Neuroscience.

The essays mirror the different approaches of their authors (mainly philosophers, but also lawyers and psychologists), therefore the book does not present a unitary and coherent view.

The editors themselves point out, in the introduction, that the "aim of the book is to deepen knowledge about neuroethics, providing the reader with a general understanding of the recent developments, highlighting the consequences deriving from these developments and introducing the terminology related to the debate and to investigation in this field". The editors choose an approach that "promotes the confrontation between reductionist and anti-reductionist views [...]; the pluralism of the views presented in the essays mirrors not just the approach preferred by each author, but also the state of the art of the debate around neuroethics, which does not allow us to close the debate giving our consensus to an approach rather than another".

Because the authors differ as to interests, approaches and competences, it is quite difficult to give a general comment on the book; I will therefore briefly introduce the main issues treated in each chapter, and I will then discuss the book in general after this rough summery of the contents.

In the first chapter (p.17-42), Andrea Lavazza addresses the question "What neuroethics is". Lavazza starts with a historical digression: from the Egyptians, to Augustine, Alberto Magnus, Descartes, Julien Offroy de la Mettrie, Pierre Broca up until Damasio and Kanwisher. Next, Lavazza introduces the development of neuroethics as a discipline which was initially dependent on neuroscience and that later developed into an independent subject in which neuroscience and philosophy, especially philosophy of mind, converge.

Lavazza introduces autonomy as a topic which seems to be particularly appropriate to neuroethical analysis: he starts from a definition of this term and then investigates the role of autonomy in particular circumstances, for instance in people affected by Alzheimer's disease, or in drugs addicts. Finally, the author briefly introduces some of the topics which are likely to keep neuroethicists busy in the next years, such as a new approach to teaching and the possible development of a new professional: "the neuro-educator". 
The second chapter (p. 43-68), authored by Michele Di Francesco (Professor of Logic and Philosophy and Science), is titled "The self between neurons and the extended mind". The author addresses the issue of the extended mind according to which the conscious mind is both inside the brain and inside the body and in the world outside the body. Di Francesco's choice to discuss the extended mind theory through "the study of conscience, does not imply an a-priori adhesion to the idea that conscience corresponds to the essence of human nature" (p.45). After introducing different definitions of conscience and the distinction between "primary conscience" and "superior conscience" (or between "nuclear conscience" and "extended conscience"), Di Francesco discusses the "internalist" and "externalist' approaches, explaining why the externalist (moderate) approach is, in his view, more plausible.

The next paragraphs explain why language, society and environment are foundational elements of the "self", coherently with an externalist approach which embraces the theory of the extended mind.

The third chapter (p. 69-83) is authored by Mario De Caro, Professor of Moral Philosophy, and it addresses the issue of free will and neuroscience. De Caro starts by introducing Libet's experiments on free will and showing how these experiments tell something about our conscience but not about our freedom. He then discusses experiments conducted by Soon et al., which followed Libet's ones. De Caro maintains that these experiments are not reliable in proving that there is not such a thing as free will, because of a number of false assumptions. For instance, the actions that the subjects were required to perform cannot be considered free choices, or even genuine choices: "the activities which are perceived as free and which, at the same time, are anticipated by the urge to perform them, are a minority and do not represent a paradigm of choices and free actions" (p.79). Moreover, according to De Caro, the results of the study by Soon et al. are compatible with all the theories of freedom, both the negative ones, such as illusionism and mysterianism, and the positive ones, such as compatibilism and libertarianism; therefore they fail to show that free will is an illusion.

Laura Boella, Professor of Moral Philosophy, is the author of the fourth chapter, which investigates the relationship between nature and morality (p.85-108). Boella introduces the classic questions about morality in the history of philosophy (what is responsibility, autonomy, freedom etc.?), suggesting that new developments in the field of neuroscience could enrich the debate about morality, but cannot substitute for theoretical analysis of these topics. The last paragraphs discuss emotions and their importance in moral decisions, and in particular the importance of "empathy" no longer considered as the origin of morality but rather as a "model of complex experiences" which includes different levels, from the "correlation between the self and others on a sensible and motorial level" to "imaginative variation" (p.106). To sum up, according to Boella, although at this stage we cannot find a linear connection between morality and neurobiological mechanisms, we can still find a bridge between culture and nature, because human capacities, like empathy, are deeply 
rooted in human nature, but can develop only when part of a cultural and social world which is rich in relationships and meanings.

The fifth chapter (p.109-134), by Antonio Da Re and Luca Grion, both Professors of Moral Philosophy, aims to discuss the relationship between the concept "person" and "neuroscience. The chapter starts by pointing out the importance of the notion of "person", but also the difficulties in providing a good definition of "person". These difficulties bring into doubt the fact that there is such a thing we can define as "person". The authors try to understand if the neurosciences can contribute in a fruitful way to the discussion about what a person is. They start from a paper by Farah and Heberlein "Personhood and Neuroscience: Naturalizing or Nihilating?" (2007) [2] which discusses the intuition according to which there is such a thing as a person, and in which the authors reject the idea that persons and non persons differ in a qualitative way. Da Re and Grion then reconstruct the debate around the naturalistic paradigm, summing up the theories of E. Phelps, P. Churchland and W. Glannon. Having introduced the "eliminativist" approach to personhood, the authors start to question the relationship between identity, mind and the brain, following arguments proposed by Dennett, but also referring to Parfit's theory about personal identity.

The authors introduce approaches against eliminativism, and propose reasons in favour of a reductionist (but not eliminativist) view, referring to arguments provided by Baker, Wiggins and Olson. The authors conclude their chapter with a general discussion of the reductionist and antireductionist paradigms, suggesting the reintroduction into the debate of the contributions of Aristotle and Thomas Aquinas, because they could provide neuroscience with fruitful hermeneutical interpretations of reality.

The sixth chapter (p. 135-164) is authored by the two editors and Luca Sammicheli (Professor of Neuropsychology). This chapter introduces the contribution that neuroethics and neurosciences are likely to make to law and justice. For instance, lie detection and memory detection are technologies which are not yet completely reliable, but which could radically change the way we deal with legal processes in the future.

Moreover, neuroscience is particularly important when we want to understand the origin of extremely violent behaviours, such as in psychopathic people. For instance, neurosciences can shed light on the relationship between intention, free will and control, clarifying the role of these elements in some extremely violent behaviours.

The authors, after clarifying the relevant terms and carefully explaining the main achievements in this field, conclude that in the future neuroethicists will have to help find a balance between the 
traditional categories of law (like responsibility, culpability and punishment) and the new elements introduced by neuroscience.

The seventh chapter (p.165-190), titled "Evolution, cognition and culture", is authored by Massimo Marraffa, Associate Professor of Logic and Philosophy of Science. The chapter starts by addressing the issues related to "relativistic culturalism", such as the difference between nature and culture and between a dualistic and a materialistic approach.

Marraffa reconstructs the history of three processes involved in the decline of "relativistic culturalism", namely: 1) the rise of cognitivistic psychology 2) the rationalistic and nativist theory of mind by Noam Chomsky and 3) the transition from socio-biology to evolutionary psychology.

These three elements, according to Marraffa, brought about the development of a cognitiveevolutioniary perspective on culture according to which cognition is a by-product of evolution. "Culture influences not just the contents of our beliefs but also the strategies we use to elaborate information about the world" (p.188). The author hopes that a look at the interactive relationship between the neuro-cognitive level and the cultural one can overcome the anachronistic approach of relativistic culturalism.

The eighth and final chapter (p.190-216) is authored by Rino Rumiati and Lorella Lotto (both Professors of psychology) and is titled "Decisions and moral decisions between rationality and emotions". The chapter aims to explain how we make moral decisions on the basis of both rational and irrational elements. The authors introduce different approaches to moral judgments; through the theories of Kohlberg, Damasio, Thomson and Greene, the authors show how emotions have been introduced in the debate on moral decisions. The authors explain, referring to a number of experiments, that moral decisions are very often influenced by the way a certain dilemma is presented to the subjects (framing effect) and that this is one of the factors that increases the level of inconsistency of moral decisions in ethical dilemmas. Rumiati and Lotto suggest that emotions involved in decision making can be "incidental" (when they are not directly related to a specific object) or "integral" (when they arise because a particular target elicits such emotions). According to the authors, we should take into account three different kinds of rationality (the authors refer to the theories of Pham, 2007 [3]): 1) the capacity to think logically 2) the coherence between actions and objectives 3) "ecological' rationality (which involves emotions and explains altruistic behaviours). The next paragraphs introduce the issue of "illusions of control" (and self-control) and "unrealistic optimism", both related to our inability to estimate our capacities and to evaluate our actions in an unbiased way (e.g. ability to drive a car or to follow a restrictive diet). The authors also discuss another element which can affect decision making: personality. Studies show that people with low conscientiousness and high extroversion are more keen on making risky choices. To conclude, Rumiati and Lotto say that both cognitive psychology and cognitive neurosciences contribute to shed light on the problem of "decision making" and that both these subjects help us to understand the role of rational and emotional elements in decisional processes and in moral judgements. 
This quick summary of the eight chapters of the book was a necessary prologue in order to introduce the main issues taken into account in the book and to give a hint of the structure of the single chapters.

As already said above, the editors clearly state in the introduction that the book does not present a unitary approach, but rather shows different views which mirror the complexity, and also the disagreements, in the current debate about neuroethics. On one hand this is certainly a positive feature, because the reader becomes aware of the contradictions, disagreements and problems of the (Italian) debate on neuroethics; but on the other hand this heterogeneous approach can generate some confusion in the reader, especially if she is not already familiar with the current debate in neuroethics.

The main problem with this book, indeed, is the fact that the target is not clearly defined; some chapters seem to be an introduction to neuroethics for a general public, whereas other ones seem to be written for an audience of experts. In particular, not all the chapters are accessible to the lay public, as they presuppose an existing basic knowledge of neuroethics and philosophy, whereas other ones would say nothing new to people who are already aware of the progress of neuroethics. In this respect, the book is quite unbalanced, with some chapters introducing concepts and ideas central to neuroethics to non-experts, and other ones just going straight to the particular aspect they mean to discuss without introducing the meaning of the concepts deployed.

Another distinctive element of the book is the historical approach to the issues discussed. In almost all the papers, the authors start with a history of a certain concept, or word, or problem. This is a very common approach to philosophy in Italy (philosophy in Italy is often history of philosophy), but a non-Italian reader would be rather puzzled by the number of quotations and references to authors like Descartes and Kant, or Augustine and Thomas Aquinas (who, obviously, were not neuroethicists) in a book about neuroethics.

The historical approach to neuroethics is also related to the heavy use of quotations of studies and theories of other neuroscientists. This is, again, a very common way to discuss philosophy in Italy, but it certainly makes it difficult to understand 1 ) what the ideas and the thesis of the single author are and 2) in what way they are different from the ones of the other authors quoted in each chapter. Also the use of the language, the structure of the sentences and the choice of the words used, are peculiar of the Italian philosophical tradition, heavily influenced by a continental approach to philosophy rather than by the analytical one (which is more commonly adopted by neuroethicists and bioethicists in the Anglo-American areas). 
This book, however, is written and published in Italian, and it is clearly meant to be read by Italians who are more familiar with the historical and continental approach to neuroethics and bioethics.

Overall Neuroetica is interesting and worth reading, not just for the specific contents of the chapters (some of them are very well written and engaging), but also because it reveals interesting differences in the way neuroethics is approached in areas influenced by different philosophical traditions.

\section{REFERENCES}

[1] Lavazza, Andrea and Sartori, Giovanni (edited by). 2011. Neuroetica, scienze del cervello, filosofia e libero arbitrio, II Mulino.

[2] Farah, Martha J., and Heberlein, Andrea S. 2007. Personhood and neuro- science: Naturalizing or nihilating? American Journal of Bioethics (AJOB-Neuroscience) 7(1): 37-48.

[3] Pham, Michel Tuam. 2007. Emotion and Rationality: A Critical Review and Interpretation of Empirical Evidence, Review of General Psychology, Vol. 11, No. 2, 155-178. 



\section{University Library}

\section{- M M N E R VA A gateway to Melbourne's research publications}

Minerva Access is the Institutional Repository of The University of Melbourne

Author/s:

Minerva, $F$

Title:

Neuroetica, a Look at the Development of the Italian Debate on Neuroethics

Date:

2013-04-01

Citation:

Minerva, F. (2013). Neuroetica, a Look at the Development of the Italian Debate on

Neuroethics. NEUROETHICS, 6 (1), pp.233-236. https://doi.org/10.1007/s12152-012-9160-x.

Persistent Link:

http://hdl.handle.net/11343/282872 